\title{
R2-IBEA: R2 Indicator Based Evolutionary Algorithm for Multiobjective Optimization
}

\author{
Dũng H. Phan \\ Department of Computer Science \\ University of Massachusetts, Boston \\ Boston, MA 02125, USA \\ Email: phdung@cs.umb.edu
}

\author{
Junichi Suzuki \\ Department of Computer Science \\ University of Massachusetts, Boston \\ Boston, MA 02125, USA \\ Email: jxs@cs.umb.edu
}

\begin{abstract}
This paper proposes and evaluates an evolutionary multiobjective optimization algorithm (EMOA) that eliminates dominance ranking in selection and performs indicator-based selection with the $\mathrm{R} 2$ indicator. Although it is known that the $\mathrm{R} 2$ indicator possesses desirable properties to quantify the goodness of a solution or a solution set, few attempts have been made until recently to investigate indicator-based EMOAs with the R2 indicator. The proposed EMOA, called R2-IBEA, is designed to obtain a diverse set of Pareto-approximated solutions by correcting an inherent bias in the $R 2$ indicator. (The R2 indicator has a stronger bias to the center of the Pareto front than to its edges.) Experimental results demonstrate that R2IBEA outperforms existing indicator-based, decomposition-based and dominance ranking based EMOAs in the optimality and diversity of solutions. R2-IBEA successfully produces diverse individuals that are distributed well in the objective space. It is also empirically verified that R2-IBEA scales well from twodimensional to five-dimensional problems.
\end{abstract}

\section{INTRODUCTION}

This paper studies an indicator-based evolutionary algorithm to solve multiobjective optimization problems (MOPs). In general, an MOP is formally described as follows.

$$
\left.\begin{array}{r}
\operatorname{minimize} F(x)=\left[f_{1}(x), f_{2}(x), \cdots, f_{m}(x)\right]^{T} \in \mathcal{O} \\
\text { subject to } x=\left[x_{1}, x_{2}, \cdots, x_{n}\right]^{T} \in \mathcal{S}
\end{array}\right\}
$$

$\mathcal{S}$ denotes the decision variable space. $x \in \mathcal{S}$ denotes a solution that consists of $n$ decision variables. It is called an individual in evolutionary multiobjective optimization algorithms (EMOAs). $F: \mathbb{R}^{n} \rightarrow \mathbb{R}^{m}$ consists of $m$ real-valued objective functions, each of which maps a solution $x$ to an objective function value (or simply, an objective value) in the objective space $\mathcal{O}$. When $m>3$, an MOP is often called highdimensional. The goal of an EMOA is to find an individual(s) that minimize(s) objective values.

In MOPs, there rarely exists a single solution that is optimum with respect to all objectives because objectives often conflict with each other. Thus, EMOAs seek the optimal tradeoff individuals, or Pareto-optimal individuals, by considering the trade-offs among conflicting objectives. The notion of dominance plays an important role to seek Pareto optimality in MOPs. An individual $x \in \mathcal{S}$ is said to dominate another individual $y \in \mathcal{S}$ (denoted by $x \succ y$ ) iif $f_{i}(\vec{x}) \leq f_{i}(\vec{y}) \forall i=$ $1, \cdots, m$ and $f_{i}(\vec{x})<f_{i}(\vec{y}) \exists i=1, \cdots, m$.
EMOAs often rank individuals based on the dominance relationships among them and exploit their ranks in selection operators. This process is called dominance ranking.

A research trend in the design of EMOAs is to adopt the notion of indicator-based selection, which augments or replaces dominance ranking with quality indicators [1]. A quality indicator measures the goodness of an individual or an individual set. Recent research findings show that indicatorbased EMOAs often outperform traditional EMOAs that use dominance ranking [2]-[6].

This paper proposes and evaluates an indicator-based EMOA, called R2-IBEA, which eliminates dominance ranking and performs selection with the $\mathrm{R} 2$ indicator [7]. The R2 indicator has been somewhat underrepresented although it possesses desirable properties [8], [9]. To the best knowledge of the authors of this paper, there exist only two lines of research efforts to study the $\mathrm{R} 2$ indicator in the context of the development of indicator-based EMOAs [8]-[11]. Along with those existing work, this paper empirically evaluates R2-IBEA with major test problems with two to five objective spaces for better understanding the impacts of the R2 indicator on the design and performance of indicator-based EMOAs.

This paper offers the following three contributions to the design space of indicator-based EMOAs:

- Hypervolume-based weight vector generation. The R2 indicator usually requires a set of weight vectors that are uniformly distributed in the objective space [8][11]. The vector generation method in R2-IBEA is designed to produce weight vectors so that they uniformly disperse and maximize their hypervolume in the objective space. It does not depend on the dimensionality of the objective space.

- A binary $R 2$ indicator. R2-IBEA leverages a binary $\mathrm{R} 2$ indicator that determines a superior-inferior relationship (or the R2 relationship) between given two individuals. This work is the first attempt to investigate a binary $\mathrm{R} 2$ indicator while a unary $\mathrm{R} 2$ indicator has been studied [8]-[11].

- Adaptive reference point adjustment. The R2 indicator often requires the fixed reference point [8]-[11]. R2IBEA dynamically adjusts the location of the reference point according to the extent of the currentgeneration individuals in the objective space. This 
adaptive method is designed to aid R2-IBEA to obtain a diverse and evenly-distributed set of individuals by correcting an inherent exploration bias in the R2 indicator. (The R2 indicator has a stronger bias to the center of the Pareto front than to its edges [9].)

Similar to existing indicator-based EMOAs [3]-[5], R2EMOA does not require a diversity preservation operator. It pursues the optimality and diversity of individuals with the $\mathrm{R} 2$ indicator.

This paper evaluates R2-EMOA with 10 well-known test problems (15 problem instances in total) with two to five objectives. Experimental results show that R2-IBEA is competitive with existing indicator-based EMOAs (IBEA- $\varepsilon 2$ [4] and R2EMOA [11]), a decomposition-based EMOA (MOEA/D [12]) and a traditional EMOA that uses dominance ranking in selection (NSGA-II [13]) and R2-IBEA often outperforms those existing EMOAs in terms of the optimality and diversity of individuals. R2-IBEA successfully obtains diverse individuals that are evenly-distributed in the objective space. It is also empirically verified that R2-IBEA scales well from twodimensional to five-dimensional problems.

\section{BACKGROUND: R2 INDICATOR}

The R2 indicator was originally proposed to assess the relative quality of two sets of individuals [7]. Assuming the standard weighted Tchebycheff function with a particular reference point $z^{*}$, the indicator can be used to assess the quality of a single individual set $(\mathcal{A})$ against $z^{*}$ [8], [9]:

$$
R 2\left(\mathcal{A}, \mathcal{V}, z^{*}\right)=\sum_{v \in \mathcal{V}}\left(p(v) \times \min _{a \in \mathcal{A}}\left\{\max _{1 \leq j \leq m} v_{j}\left|z_{j}^{*}-a_{j}\right|\right\}\right)
$$

$\mathcal{V}$ denotes a set of weight vectors. Each weight vector $v=\left(v_{1}, \ldots, v_{m}\right) \in \mathcal{V}$ is placed in the $m$-dimensional objective space. $p$ denotes a probability distribution on $\mathcal{V}$. Weight vectors are often chosen uniformly distributed in the objective space [8]-[11]. In this case, the R2 indicator is described as:

$$
R 2\left(\mathcal{A}, \mathcal{V}, z^{*}\right)=\frac{1}{|\mathcal{V}|} \sum_{v \in \mathcal{V}} \min _{a \in \mathcal{A}}\left\{\max _{1 \leq j \leq m} v_{j}\left|z_{j}^{*}-a_{j}\right|\right\}
$$

A utopian point is usually used as the reference point $z^{*}[8]-[11]$. A utopian point is a point that is never dominated by any feasible solutions in the objective space. For example, it is $(0,0)$ in a two-dimensional objective space where each objective value is greater than or equal to 0 .

A lower $\mathrm{R} 2$ value indicates that an individual set $\mathcal{A}$ is closer to the reference point. $R 2\left(\{x\}, \mathcal{V}, z^{*}\right)=0$ when an individual $x \in \mathcal{S}$ is positioned on the reference point.

The R2 indicator possesses a desirable property of weak monotonicity. When an individual $x \in \mathcal{S}$ dominates another individual $y \in \mathcal{S}$ (i.e., $x \succ y), R 2\left(\{x\}, \mathcal{V}, z^{*}\right) \leq R 2\left(\{y\}, \mathcal{V}, z^{*}\right)$.

\section{RELATED WORK}

The R2 indicator was originally proposed in [7], together with two other variants: the R1 and R3 indicators. The R2 indicator is recommended as one of the best quality indicators in [8], along with the hypervolume (HV) indicator [14] and the $\varepsilon 2$ indicator [4]. $\mathrm{HV}$ is known to be the only indicator that preserves the property of strict monotonicity; i.e. $H V(\{x\})>$ $H V(\{y\})$ if $x \succ y$. Although the $\mathrm{R} 2$ indicator is weakly monotonic, it is computationally much less expensive than the hypervolume indicator. The computational cost to compute $\mathrm{HV}$ values grows exponentially as the number of objectives increases [15]. The $\varepsilon 2$ indicator is weak monotonic as well [4].

The HV indicator and its variants are used in several indicator-based EMOAs [3], [5], [10], [16]. For example, SMS-EMOA uses dominance ranking as the first criterion in selection and a variant of the $\mathrm{HV}$ indicator as the second selection criterion [3]. SPAM uses a unary R2 indicator, which is described in Equation 3, as well as the $\mathrm{HV}$ indicator in selection [10]. In contrast, R2-IBEA avoids the HV indicator due to its high computational cost and uses a binary R2 indicator as the sole criterion in selection.

R2-EMOA extends SMS-EMOA by replacing the HV indicator with a unary R2 indicator (Equation 3) [11]. It is computationally less expensive than SMS-EMOA; however, it still uses dominance ranking as the first criterion in selection. In contrast, R2-IBEA never relies on dominance ranking but leverages a binary $\mathrm{R} 2$ indicator.

SPAM and R2-EMOA use the fixed utopian point as the reference point ( $z^{*}$ in Equation 3 ) to compute R2 values. Unlike them, R2-IBEA dynamically adjusts the location of the reference point in each generation for attracting individuals to extreme regions in the objective space.

IBEA avoids dominance ranking and uses a binary $\varepsilon$ indicator (or a binary HV indicator) in selection [4]. IBEA is similar to R2-IBEA in that both EMOAs eliminate dominance ranking with a binary indicator. This work can be viewed as an extension of IBEA with a binary R2 indicator and its associated methods (i.e., weight vector generation and reference point adjustment methods).

MOEA/D is similar to R2-IBEA in that both EMOAs use the Tchebycheff function with uniformly distributed weight vectors [12]. It decomposes an MOP into multiple scalar optimization sub-problems. Each sub-problem corresponds to a single Tchebycheff function. MOEA/D intends to optimize multiple Tchebycheff function values while R2-IBEA aggregates multiple Tchebycheff function values into a single (R2) value and strives to optimize the aggregated value.

\section{R2-IBEA: R2 INDICATOR BASED EVOLUTIONARY ALGORITHM}

This section describes a method for weight vector generation and discuss R2-IBEA's algorithmic structure and its operators.

\section{A. Hypervolume-based Weight Vector Generation}

Algorithm 1 shows a method that R2-EMOA performs to generate uniformly distributed weight vectors $\mathcal{V}$ (c.f. Equation 3 in Section III). This process is executed offline only 


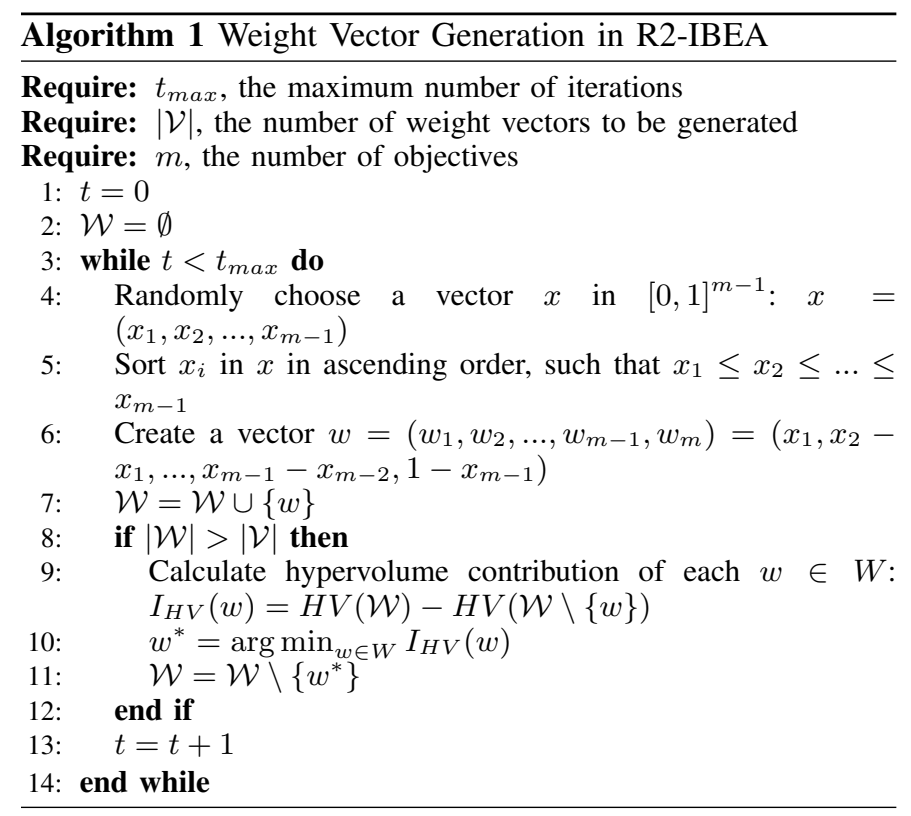

once. The objective space is assumed to be normalized into $[0,1]$ in each dimension.

At each iteration, a vector $x=\left(x_{1}, x_{2}, \ldots, x_{m-1}\right)$ is randomly chosen in $[0,1]^{m-1}$, following a uniform distribution (Line 4 in Algorithm 1). Then an $m$-dimensional vector $w$ is created with $x_{1}, x_{2}, \ldots, x_{m-1}$ (Lines 5 and 6). Note that $\sum_{i=1}^{m} w_{i}=1$. Since the mapping from $x$ to $w$ is linear, the uniformity of distribution is preserved. As described in Line 8 to 12 , the proposed method generates more vectors than $|\mathcal{V}|$ (i.e., the required number of vectors) and removes excess based on each vector's hypervolume contribution $\left(I_{H V}(w)\right.$ in Algorithm 1, Line 9). It is designed to produce weight vectors that uniformly disperse and maximize their hypervolume in the objective space. The hypervolume indicator is used because it can quantify the distribution of given vectors. In [17], it is proved that, for a linear front, the hypervolume of vectors is maximized when they are uniformly distributed.

The proposed method does not depend on the dimensionality of the objective space. It works in the same way for low-dimensional to high-dimensional spaces, unlike an angular method in [9]. Also, it can generate an arbitrary number of weight vectors $(|\mathcal{V}|)$ in any dimensional spaces while a coordinate-based method in [9], [12] cannot in three or higher dimensional spaces. For example, in a three-dimensional space, the method in [9], [12] can generate 91, 105 and 120 vectors but cannot generate 92 to 104 and 106 to 119 vectors. In a five-dimensional space, it can generate 35,70 and 126 vectors but cannot generate 36 to 69 and 71 to 125 vectors.

Figure 1 show the weight vectors generated by using the proposed method with $|\mathcal{V}|=100$ and $t_{\max }=10,000$. $(2.0,2.0)$ is used as the reference point to compute hypervolume values.

\section{B. Binary $R 2$ Indicator}

R2-IBEA performs parent selection and environmental selection with a binary R2 indicator $\left(I_{R 2}\right)$ :

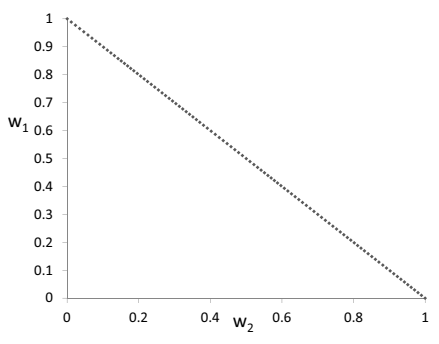

(a) Vectors in $2 \mathrm{D}$ space

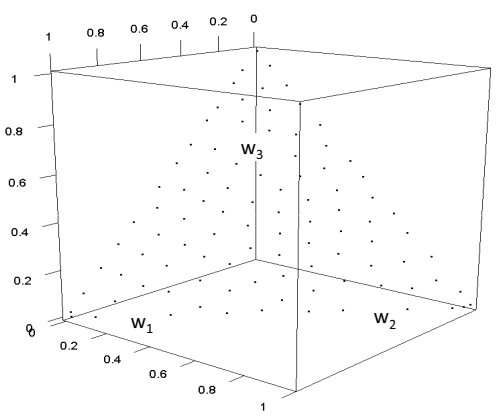

(b) Vectors in 3D space

Fig. 1: Generated Weight Vectors

$$
I_{R 2}(x, y)=R 2\left(\{x\}, \mathcal{V}, z^{*}\right)-R 2\left(\{x \cup y\}, \mathcal{V}, z^{*}\right)
$$

$I_{R 2}$ is designed to determine a superior-inferior relationship between given two individuals ( $x$ and $y$ ) with two R2 values. Each R2 value is obtained with Equation 3. If $x \succ y$, $I_{R 2}(x, y)=0$; otherwise, $I_{R 2}(x, y) \geq 0$. Given the property of weak monotonicity:

$$
\begin{array}{ll}
\text { - } & I_{R 2}(x, y) \leq I_{R 2}(y, x) \text { if } x \succ y \\
\text { - } & I_{R 2}(x, y) \geq I_{R 2}(y, x) \text { if } y \succ x
\end{array}
$$

\section{R2-IBEA's Algorithmic Structure}

Algorithm 2 shows R2-IBEA's algorithmic structure, which extends IBEA [4], an existing indicator-based EMOA.

In the 0 -th generation $(g=0), \mu$ individuals are randomly generated as the initial population $\mathcal{P}_{0}$ (Line 2). In each generation $(g)$, a pair of individuals, called parents $\left(p_{1}\right.$ and $p_{2}$ ), are chosen from the current population $\mathcal{P}_{g}$ with a binary tournament operator (Lines 6 and 7). This operator randomly draws two individuals from $\mathcal{P}_{g}$, determines the $\mathrm{R} 2$ relationship between them with the binary R2 indicator described in Section IV-B, and selects a superior one as a parent. If the two individuals yield the same $I_{R 2}$ value, one of them is selected as a parent at random.

With the crossover rate $P_{c}$, two parents reproduce two offspring with the SBX (self-adaptive simulated binary crossover) operator [18] (Line 9). Polynomial mutation [13] is performed on each offspring with the mutation rate $P_{m}$ (Lines 10 to 15). 


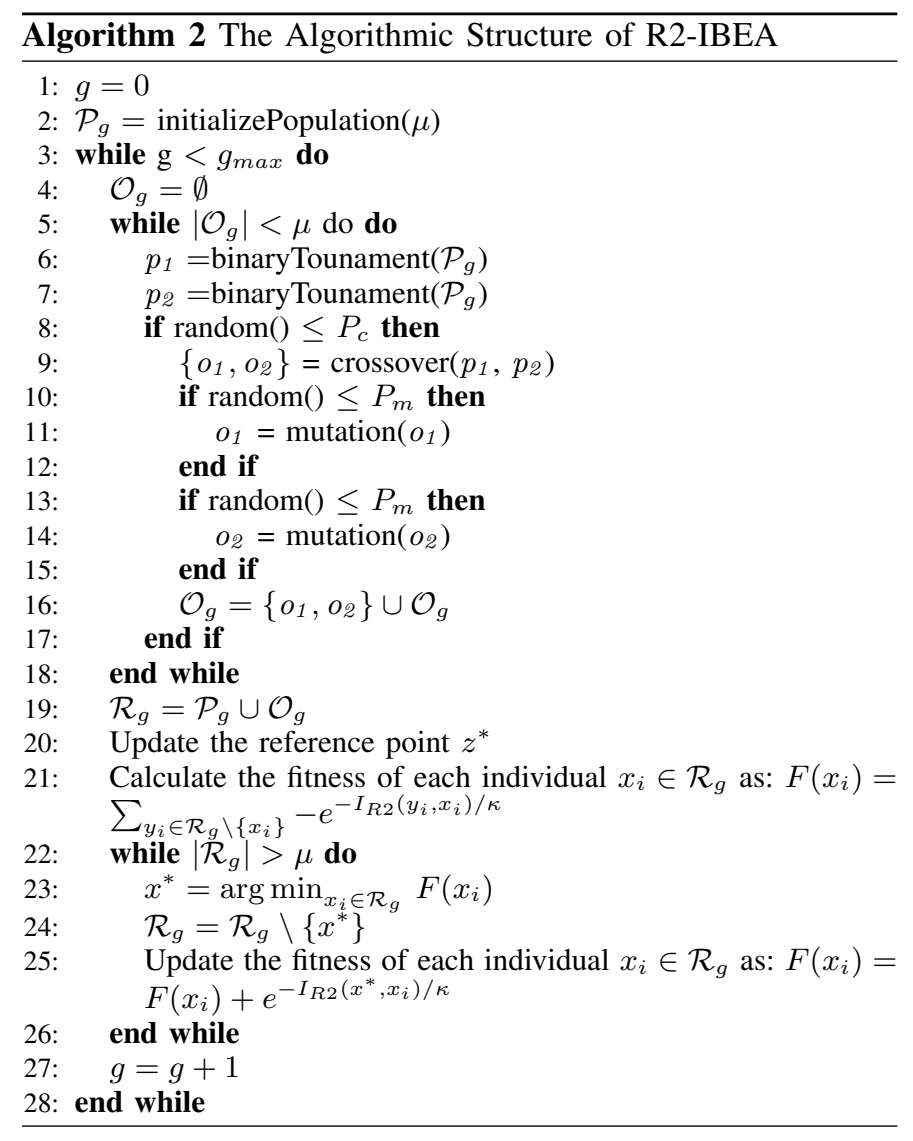

Parent selection, crossover and mutation operators are repeatedly executed on $\mathcal{P}_{g}$ until $\mu$ offspring are reproduced (i.e., until $\left|O_{g}\right|=\mu$ ). The offspring $\left(\mathcal{O}_{g}\right)$ are combined with the population $\mathcal{P}_{g}$ to form $\mathcal{R}_{g}\left(\left|R_{g}\right|=2 \mu\right)$, which is a pool of candidates for the next-generation individuals (Line 19).

Environmental selection follows offspring reproduction (Line 21 to 26). In Line 21 the fitness of each individual in $\mathcal{R}_{g}$ is calculated by applying the individual's $I_{R 2}$ value to an exponential amplification function. Then, the worst individual (i.e., the one with the lowest fitness) is removed from $\mathcal{R}_{g}$ (Lines 23 and 24). In Line 25, fitness is recalculated for each of the remaining individuals in $\mathcal{R}_{g}$. By repeating this removal process until $\left|\mathcal{R}_{g}\right|=\mu$, R2-IBEA selects $\mu$ individuals from $\mathcal{R}_{g}$ as the individuals to be used in the next generation $(g+1)$.

\section{Adaptive Reference Point Adjustment}

As described in Section II, the R2 indicator requires the reference point $\left(z^{*}\right.$ in Equation 2). All existing R2-based EMOAs use the utopian point as the reference point [9], [11]. Given this location choice for the reference point, they often fail to obtain extreme individuals particularly in the problems that have convex Pareto fonts because the R2 indicator has an inherent bias toward the center of a Pareto front [9].

In order to overcome this problem, R2-IBEA determines the reference point so that it is far enough from the individuals in the current population. It can be positioned even in the infeasible region in the objective space. This is intended to increase the density of weight vectors in extreme regions in the feasible objective space. This density increase can contribute to attract more extreme individuals.

R2-IBEA dynamically adjusts the coordinate of the reference point $z^{*}=\left(z_{1}^{*}, z_{2}^{*}, \ldots, z_{m}^{*}\right)$ as follows:

$$
z_{i}^{*}=\min _{x \in \mathcal{R}_{g}}\left\{f_{i}(x)\right\}-\max _{j=1, . ., m}\left\{\max _{x \in \mathcal{R}_{g}}\left\{f_{j}(x)\right\}-\min _{x \in \mathcal{R}_{g}}\left\{f_{j}(x)\right\}\right\}
$$

This location adjustment is carried out in every generation according to the extent of the individuals in $\mathcal{R}_{g}$ in the objective space. (See also Line 20 in Algorithm 2.)

Figure 2 illustrates an example selection of the reference point $\left(z^{*}\right)$ when $\left|\mathcal{R}_{q}\right|=6$. The coordinate of $z^{*}$ is computed with the extent of those six individuals over the horizontal objective $(b)$ and the vertical objective $(a)$.

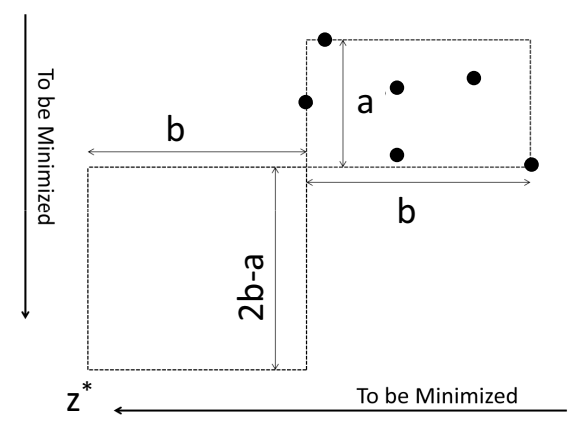

Fig. 2: An Example Reference Point Selection $(b>a)$

\section{EXPERIMENTAL RESULTS}

This section evaluates R2-IBEA with five ZDT family problems [19] and five DTLZ family problems [20], and compares R2-IBEA with existing EMOAs: IBEA- $\varepsilon 2$ [4], MOEA/D [12], R2-EMOA [11] and NSGA-II [13]. IBEA$\varepsilon 2$ is an indicator-based EMOA that uses the $\varepsilon 2$ indicator in selection. NSGA-II is one of the most classical EMOAs, which uses dominance ranking in selection.

\section{A. Experimental Configurations}

R2-IBEA was configured as shown in Table I. IBEA- $\varepsilon 2$, MOEA/D, R2-EMOA and NSGA-II were configured as described in [4], [12], [11] and [13], respectively. The population size $\mu$ is fixed to 100 in all EMOAs for all problems. The number of weight vectors $(|\mathcal{V}|)$ is set to be equal to $\mu$ in R2IBEA, R2-EMOA and MOEA/D for all problems. The number of objective function evaluations in each experiment is 50,000 for DTLZ3 and 30,000 for the other problems. This means that the number of generations in an experiment $\left(g_{\max }\right)$ is 500 for DTLZ3 and 300 for the other problems. All experiments were conducted with jMetal [21]. Each experimental result is the average of 20 independent results.

Each ZDT problem has two objectives. ZDT1, ZDT2 and ZDT3 have 30 decision variables each. ZDT4 and ZDT6 have 10 decision variables each. This experimental study 
uses three-objective and five-objective DTLZ problems. When three objectives are used, DTLZ1, DTLZ2, DTLZ3, DTLZ4 and DTLZ7 have 7, 12, 12, 12 and 22 decision variables, respectively. When five objectives are used, they have 9, 14, 14,14 and 24 decision variables, respectively. These are the default settings in jMetal.

TABLE I: Experimental Configurations

\begin{tabular}{|c|c|}
\hline Parameter & Value \\
\hline \hline$z^{*}$ (Algorithm 1) & $(2.0,2.0)$ \\
$\mathcal{V}$ (Equation 3) & 100 \\
$\mu$ (Algorithm 2) & 100 \\
$P_{c}$ (Algorithm 2) & 0.9 \\
$P_{m}$ (Algorithm 2) & $1 /$ (\# of decision variables) \\
$\kappa$ (Algorithm 2) & 0.005 \\
$g_{\max }$ (Algorithm 2) & 300 (all problems except DTLZ3) \\
& 500 (DTLZ3) \\
\hline
\end{tabular}

\section{B. Evaluation Metrics}

This paper uses the following evaluation metrics: hypervolume ratio (HVR), generalized spread (GS), generational distance (GD), inverted generational distance (IGD) and epsilon metrics.

HVR is calculated as the ratio of the hypervolume ( $H V$ ) of non-dominated individuals $(D)$ to the hypervolume of Paretooptimal solutions $\left(P^{*}\right)$ [22].

$$
H V R=\frac{H V(D)}{H V\left(P^{*}\right)}
$$

$H V$ is the union of the volumes that non-dominated individuals dominate [14]. (It is computed with the reference point whose coordinate consists of the maximum objective values.) Thus, HVR quantifies the optimality and diversity of non-dominated individuals. A higher HVR indicates that nondominated individuals are closer to the Pareto front and more diverse in the objective space.

GS is computed as follows:

$$
G S\left(D, P^{*}\right)=\frac{\sum_{i=1}^{m} d\left(e_{i}, D\right)+\sum_{x \in P^{*}}|d(x, D)-\bar{d}|}{\sum_{i=1}^{m} d\left(e_{i}, D\right)+\left|P^{*}\right| \bar{d}}
$$

$e_{1}, \ldots, e_{m}$ are $m$ extreme individuals in $P^{*}$; i.e., $e_{i}$ is the individual that yields the best result in $D$ with respect to the $i$-th objective. $d(x, D)$ is the minimum distance from an individual $x$ to the closest individual in $D \cdot \bar{d}=\frac{1}{\left|P^{*}\right|} \sum_{x \in P^{*}} d(x, D)$

GS measures how evenly non-dominated individuals are distributed in the objective space [23]. A lower GS indicates that non-dominated individuals are distributed in the objective space more evenly. GS is an extension to the spread metric, which can be used in two dimensional problems only [13]. In contrast, GS can be used in the problems with more than two objectives.

GD is computed as follows.

$$
G D=\sum_{i=1}^{|D|} \frac{d\left(d_{i}, P^{*}\right)}{|D|}
$$

$d\left(d_{i}, P^{*}\right)$ denotes the minimum distance from a nondominated individual $d_{i}$ to the closest Pareto-optimal solution in the objective space [24]. GD measures the optimality of non-dominated individuals. A lower GD indicates that nondominated individuals are closer to the Pareto-optimal front.

IGD is computed as follows where $d\left(v_{i}, D\right)$ denotes the minimum distance from a Pareto-optimal solution $v_{i}$ to the closest non-dominated individual in the objective space [24].

$$
I G D=\sum_{i=1}^{\left|P^{*}\right|} \frac{d\left(v_{i}, D\right)}{\left|P^{*}\right|}
$$

IGD measures the optimality and diversity (more specifically, the extent) of non-dominated individuals. A lower IGD indicates that non-dominated individuals are closer to the Pareto-optimal front and their extent is wider.

The epsilon metric is computed as follows [25]:

$$
\text { Epsilon }=\inf f_{\epsilon}\left\{\forall y \in P^{*}, \exists x \in D: y_{i} \geq x_{i}-\epsilon \forall i\right\}
$$

It represents the minimum distance ( $\epsilon$ value) by which each individual in $D$ can be translated in each dimension such that the Pareto-optimal solutions $P^{*}$ are weakly dominated.

For both HVR, GS, IGD and Epsilon metrics, $P^{*}$ are taken uniformly from the Pareto-optimal front. $\left|P^{*}\right|=1,001,1,001$, $269,1,001,1,001,10,000,10,000,4,000,4,000$ and 676 in ZDT1, ZDT2, ZDT3, ZDT4, ZDT6, DTLZ1, DTLZ2, DTLZ3, DTLZ4 and DTLZ7. This is the default setting in jMetal.

\section{Evaluation of Adaptive Reference Point Selection}

This section evaluates R2-IBEA's adaptive reference point selection described in Section IV-D. Figure 3 shows the individuals that R2-IBEA produces at the last generation with and without adaptive reference point selection in ZDT4, DTLZ1 and DTLZ7. (All individuals in the population are nondominated at the last generation.) ZDT4, DTLZ1 and DTLZ7 have a continuous and convex Pareto front, a continuous and linear Pareto front and discontiguous and convex Pareto fronts, respectively. When adaptive reference point selection is disabled, R2-IBEA uses a Utopian point, $(0,0)$ or $(0,0,0)$, as the fixed reference point in every generation throughout an experiment. All the other settings are identical between the two configurations of R2-IBEA.

Figure 3 confirms that the R2 indicator favors balanced individuals over extreme ones, as stated in [9], when it uses a Utopian point as the reference point. Without adaptive reference point selection, R2-IBEA fails to obtain extreme individuals at the edges of a Pareto front (Figures 3a, 3c and $3 \mathrm{e}$ ). In contrast, R2-IBEA successfully explores the edges of a Pareto front and obtains both balanced and extreme individuals when it uses adaptive reference point selection (Figures 3b, 3d and 3f). Moreover, the distribution of individuals is more even, compared to the cases with adaptive reference point selection disabled. Figure 3 demonstrates that adaptive reference point selection effectively corrects an inherent bias 
of the R2 indicator toward the center of a Pareto front and aids R2-IBEA to obtain a diverse and evenly-distributed set of Pareto-approximated individuals.

\section{Comparative Evaluation of R2-IBEA}

This section compares R2-IBEA with four existing EMOAs in terms of the optimality and diversity of individuals. Table II shows the average HVR, GS, GD, IGD and epsilon values that R2-IBEA and four other EMOAs yield at the last generation in two-dimensional ZDT problems. A number in parentheses indicates a standard deviation among 20 experiments. A bold number indicates the best result among five EMOAs. A star symbol (*) and a sharp symbol (\#) are placed when a result is statistically different from R2-IBEA's result based on a single-tail $t$-test or a Wilcoxon Mann Whitney (WMW) test, respectively. The significance level of $95 \%$ is used in both tests.

As shown in Table II, R2-IBEA yields the best HVR results in three of five ZDT problems and significantly outperforms all the other EMOAs with the significance level of $95 \%$ in both $t$-test and WMW test. In GS, R2-IBEA performs best in three of five ZDT problems. It yields the best GD value in a ZDT problem, the best IGD results in two ZDT problems, and the best epsilon values in three ZDT problems. In these problems, R2-EMOA significantly outperforms the other EMOAs in both $t$-test and WMW test.

Table III shows the HVR, GS, GD, IGD and epsilon results in three-dimensional DTLZ problems. R2-IBEA yields the best HVR and GS results in all DTLZ problems. In these two metrics, R2-IBEA significantly outperforms four other EMOAs in both $t$-test and WMW test. This superiority in HVR and GS is consistent with the observation that Section V-C made for Figures 3d and 3f. In GD and IGD, R2-IBEA outperforms four other EMOAs in three DTLZ problems. R2-IBEA yields the best epsilon values in four DTLZ problems. In these problems, it significantly outperforms four other EMOAs in both $t$-test and WMW test.

In HVR and IGD, R2-IBEA significantly outperforms IBEA- $\varepsilon 2$, in both $t$-test and WMW test, in all ZDT and DTLZ problems except ZDT1. In GS, R2-IBEA significantly outperforms IBEA- $\varepsilon 2$, in both $t$-test and WMW test, in all ZDT and DTLZ problems. In comparison to R2-EMOA, R2-IBEA yields better HVR and IGD results in all ZDT and DTLZ problems. R2-IBEA significantly outperforms R2-EMOA, in WMW test, in all the problems. In GS, R2-IBEA outperforms $\mathrm{R} 2$-EMOA, in both $t$-test and WMW test, in all problems except ZDT3. These results demonstrate that R2-IBEA is competitive with existing indicator-based EMOAs and often yields superior performance over those existing EMOAs in terms of both optimality and diversity.

In comparison to NSGA-II, R2-IBEA yields significantlybetter HVR results, in both $t$-test and WMW test, in all ZDT and DTLZ problems except ZDT3. In GS, R2-IBEA significantly outperforms NSGA-II, in WMW test, in all ZDT and DTLZ problems except ZDT3. It yields significantly-better GD and epsilon results, in both $t$-test and WMW test, in eight of ten problems. In IGD, R2-IBEA outperforms NSGAII, in both $t$-test and WMW test, in five of the problems.
These results demonstrate that R2-IBEA maintains superior performance over NSGA-II in both optimality and diversity.

Table IV shows the hypervolume (HV) results in fivedimensional DTLZ problems. Due to lack of Pareto-optimal solutions, HV is used instead of HVR. For the same reason, GS, GD, IGD and epsilon metrics are not used. As illustrated in Table IV, R2-IBEA significantly outperforms four other EMOAs, in WMW test, in four of five problems. In DTLZ2, IBEA- $\epsilon 2$ and R2-IBEA yield the best and second best HV values, respectively. Note that R2-IBEA is more stable than four other EMOAs; it constantly yields competitive results in all five-dimensional DTLZ problems while other EMOAs perform well in some problems and poorly in other problems. For example, IBEA- $\epsilon 2$ yields the best HV value in DTLZ2; however, its HV value is very low in DTLZ3. Tables II, III and IV demonstrate that R2-IBEA scales better than four other EMOAs from two-dimensional to five-dimensional problems.

It is noticeable in Tables II and III that standard deviations are relatively high in ZDT4, DTLZ3 and DTLZ4. Therefore, Figure 4 shows the boxplots for those problems in order to examine the degree of dispersion and skewness in HVR, GS, GD and IGD. A box in each boxplot contains the middle $50 \%$ of individuals. The upper edge of the box indicates the 75 th percentile of individuals, and the lower edge indicates the 25th percentile. The middle horizontal line in the box indicates the 50th percentile (i.e., the median). The ends of a vertical line indicate the minimum individual within $1.5 \times \mathrm{IQR}$ (interquartile range) of the lower quartile and the maximum individual within $1.5 \times \mathrm{IQR}$ of the upper quartile.

In ZDT4 and DTLZ3, R2-IBEA yields the best median results and the minimum dispersion of individuals among five EMOAs in all four metrics. In DTLZ4, R2-IBEA yields the best median results in HVR, GS and IGD. The dispersion of R2-IBEA individuals is minimum in HVR, GD and IGD. Except for GS in DTLZ7, R2-IBEA achieves competitive (often the best) performance among five EMOAs in median, IQR and $1.5 \times$ IQR metrics although it tends to produces a few outliers in DTLZ4.

\section{CONCLUSIONS}

This paper investigates an indicator-based EMOA, called R2-IBEA, which leverages the R2 indicator in selection instead of traditional dominance ranking. R2-IBEA uses a binary $\mathrm{R} 2$ indicator that determines a superior-inferior relationship between given two individuals by adaptively adjusting the location of the reference point according to the extent of the current-generation individuals in the objective space. Experimental results show that R2-IBEA outperforms existing indicator-based, decomposition-based and dominance ranking based EMOAs in the optimality and diversity of solutions. R2-IBEA scales better than those existing EMOAs from twodimensional to five-dimensional problems. It is also verified that R2-IBEA successfully produces a diverse set of individuals that are evenly distributed in the objective space.

Several future work is planned. R2-IBEA is planned to be evaluated in more details; for example, with different settings for parameters such as $\mathcal{V}$ and $\kappa$ (Table I). More comprehensive comparative study is planned as well. In addition, other indicators than the hypervolume indicator will be considered 
TABLE II: Comparison of R2-IBEA with Four Other EMOAs in ZDT problems (Two Objectives). * and \# are placed when a result is statistically different from R2-IBEA's result based on a $t$-test or a WMW test, respectively.

\begin{tabular}{|c|c|c|c|c|c|c|}
\hline Problem & Algorithm & HVR & GS & GD & IGD & Epsilon \\
\hline \multirow{5}{*}{ ZDT1 } & "R2-IBEA & $0.99285(4.1 \mathrm{E}-4)$ & $\overline{0.27544}(0.02559)$ & 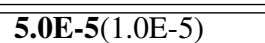 & $1.9 \mathrm{E}-4(3.0 \mathrm{E}-5)$ & $0.00976(0.00258)$ \\
\hline & NSGAII & $0.99032(4.2 \mathrm{E}-4)^{*} \#$ & $0.38279(0.03301)^{* \#}$ & $2.1 \mathrm{E}-4(3.0 \mathrm{E}-5)^{* \#}$ & $1.9 \mathrm{E}-4(1.0 \mathrm{E}-5)$ & $0.01409(0.0031)^{* \#}$ \\
\hline & MOEA/D & $0.99151(4.0 \mathrm{E}-4) * \#$ & $0.29222(0.01406)^{* \#}$ & $1.2 \mathrm{E}-4(3.0 \mathrm{E}-5)^{* \#}$ & $1.7 \mathrm{E}-4(1.0 \mathrm{E}-5)$ & $0.01112(0.00313)$ \\
\hline & IBEA- $\varepsilon 2$ & $0.99329(1.1 \mathrm{E}-4)^{* \#}$ & $0.2758(0.03273)$ & $7.0 \mathrm{E}-5(3.0 \mathrm{E}-5)^{* \#}$ & $1.5 \mathrm{E}-4(0.0)^{* \#}$ & E-4)*\# \\
\hline & R2-EMOA & $0.98633(0.01287) * \#$ & $0.51993(0.06544)^{* \#}$ & $1.7 \mathrm{E}-4(4.0 \mathrm{E}-5)^{* \#}$ & $5.7 \mathrm{E}-4(0.00109) \#$ & $0.03307(0.04692)^{* \#}$ \\
\hline \multirow{5}{*}{ ZDT2 } & $\begin{array}{l}\text { R2-IBEA } \\
\end{array}$ & $\overline{\mathbf{0 . 9 8 6 6 2}(2.0 \mathrm{E}-4)}$ & $\overline{\mathbf{0 . 2 2}(0.03036)}$ & 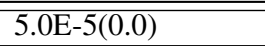 & $2.8 \mathrm{E}-4(3.0 \mathrm{E}-5)$ & $\begin{array}{l}\mathbf{0 . 0 0 7 2 4}(5.2 \mathrm{E}-4) \\
\end{array}$ \\
\hline & NSGAII & $0.98131(6.9 \mathrm{E}-4) * \#$ & $0.39562(0.04495)^{* \#}$ & $1.6 \mathrm{E}-4(3.0 \mathrm{E}-5)^{* \#}$ & $1.9 \mathrm{E}-4(1.0 \mathrm{E}-5)^{* \#}$ & $0.01267(0.00153)^{* \#}$ \\
\hline & MOEA/D & $0.98336(0.00105) * \#$ & $0.22738(0.05508)$ & $1.0 \mathrm{E}-4(3.0 \mathrm{E}-5)^{* \#}$ & 1.5E-4 $(1.0 \mathrm{E}-5) * \#$ & $0.00879(0.00203)^{* \#}$ \\
\hline & IBEA- $\varepsilon 2$ & $0.98385(3.7 \mathrm{E}-4) * \#$ & $0.42629(0.02901) * \#$ & $4.0 \mathrm{E}-5(0.0)^{*} \#$ & $4.1 \mathrm{E}-4(4.0 \mathrm{E}-5) * \#$ & $(0.00165) * \#$ \\
\hline & R2-EMOA & $0.78053(0.2073) * \#$ & 0.77447 & $4.0 \mathrm{E}-5$ & $0.00503(0.00449)^{* \#}$ & $0.38324(0.30858) * \#$ \\
\hline \multirow{5}{*}{ ZDT3 } & R2-IBEA & 0.996 & $\overline{0.690}$ & $\bar{~} 1.2 \mathrm{E}-4$ & $\overline{7.2 \mathrm{E}-4}$ & $\overline{06903)}$ \\
\hline & NSGAII & 0.99642 & $0.39401(0.04603)^{* \#}$ & $2.1 \mathrm{E}-4(1.0 \mathrm{E}-5)^{* \#}$ & 2.6E-4(1.0E-5)\# & $\mathbf{0 . 0 0 8 7 6}(0.00156) \#$ \\
\hline & MOEA/D & $0.99281(2.7 \mathrm{E}-4)^{* \#}$ & $0.88724(0.03942)^{* \#}$ & $1.8 \mathrm{E}-4(1.0 \mathrm{E}-5)^{* \#}$ & $5.6 \mathrm{E}-4(1.0 \mathrm{E}-5) \#$ & $0.01546(2.5 \mathrm{E}-4) \#$ \\
\hline & IBE & $\mathrm{E}-4)^{* \#}$ & 0.808 & $1.2 \mathrm{E}-6$ & $0.00186(6.0 \mathrm{E}-5)^{* \#}$ & 132)\# \\
\hline & R2-EMOA & $0.98903(0.00389)^{* \#}$ & $0.50758(0.07712)^{* \#}$ & $1.8 \mathrm{E}-4(2.0 \mathrm{E}-5)^{* \#}$ & $0.00143(0.002) \#$ & $0.07941(0.12471) \#$ \\
\hline \multirow{5}{*}{ ZDT4 } & R2-IBEA & $\mathbf{0 . 9 8 9 8}(0.00265)$ & 0.5095 & 0.0280 & $1.7 \mathrm{E}-4(2.0 \mathrm{E}-5)$ & $\mathbf{0 . 0 0 9 4}(0.00152)$ \\
\hline & NSGAII & $0.98511(0.00272) * \#$ & 0.399 & $3.7 \mathrm{E}-4$ & $2.1 \mathrm{E}-4(2.0 \mathrm{E}-5) * \#$ & 0.013 \\
\hline & MOEA/D & $(0.00559) * \#$ & 0.506 & 00228)\# & $2.8 \mathrm{E}-4(6.0 \mathrm{E}-5) * \#$ & $00344)^{* \#}$ \\
\hline & IBEA- $\varepsilon 2$ & $0.79795(0.09022) * \#$ & $1.22203(0.06738)^{* \# \#}$ & $5.1 \mathrm{E}-4(6$ & $0.00993(0.00397) * \#$ & $0.35924(0.12693) * \#$ \\
\hline & R2-EMOA & $0.8775(0.05538) * \#$ & $0.06267)^{* \#}$ & 3.3E-4 & $0.00709(0.00267)^{* \#}$ & $0.27767(0.08634)^{* \#}$ \\
\hline \multirow{5}{*}{ ZDT6 } & R2-IBEA & 0.992 & 0.257 & $\overline{5.6 \mathrm{E}-4}$ & $1.4 \mathrm{E}-4$ & \\
\hline & NSGAII & $0.97976(0.00234) * \#$ & $0.362(0.03474)^{* \#}$ & $7.2 \mathrm{E}-4(5.0 \mathrm{E}-5)^{* \#}$ & $2.3 \mathrm{E}-4(3.0 \mathrm{E}-5)^{* \#}$ & $0.01196(0.00131)^{* \#}$ \\
\hline & MOEA/D & $498)^{* \#}$ & 4414)*\# & $3 \mathrm{E}-4)^{*} \#$ & $4.2 \mathrm{E}-4(7.0 \mathrm{E}-5) * \#$ & $0.01624(0.00272)^{*} *$ \\
\hline & IBEA- $\varepsilon 2$ & $.00136)^{* \# \#}$ & $0.70287(0.04334)^{* \#}$ & 4.5E-4(3.0E-5)*\# & $1.7 \mathrm{E}-4(1.0 \mathrm{E}-5)^{* \#}$ & $0.0101(0.00121)^{* \#}$ \\
\hline & R2-EMOA & $0.98343(0.00163) * \#$ & $0.46308(0.04175)^{* \#}$ & $6.4 \mathrm{E}-4(3.0 \mathrm{E}-5)^{* \#}$ & $2.6 \mathrm{E}-4(5.0 \mathrm{E}-5)^{* \#}$ & $0.01258(0.00164)^{* \#}$ \\
\hline
\end{tabular}

and evaluated for R2-IBEA's weight vector generation method (Algorithm 1).

\section{REFERENCES}

[1] C. C. Coello, "Evolutionary multi-objective optimization: Some current research trends and topics that remain to be explored," Front. Computat. Sci. China, vol. 3, no. 1, 2009.

[2] T. Wagner, N. Beume, and B. Naujoks, "Pareto-, aggregation-, and indicator-based methods in many-objective optimization," in Proc. Int'l Conf. Evol. Multi-criterion Optimization, 2007.

[3] N. Beume, B. Naujoks, and M. Emmerich, "SMS-EMOA: Multiobjective selection based on dominated hypervolume," Eur. J. Oper. Res. vol. 181, no. 3, pp. 1653-1669, Sept. 2007.

[4] E. Zitzler and S. Künzli, "Indicator-based selection in multiobjective search," in Proc. Int'l Conf. on Parall. Prob. Solving from Nature, 2004.

[5] D. H. Phan, J. Suzuki, and P. Boonma, "SMSP-EMOA: Augmenting SMS-EMOA with the prospect indicator for multiobjective optimization," in Proc. of IEEE Int'l Conf. on Tools with Artif. Int., 2011.

[6] D. H. Phan and J. Suzuki, "Leveraging indicator-based ensemble selection in evolutionary multiobjective optimization algorithms," in Proc. of ACM Int'l Genetic and Evol. Computat. Conference, 2012.

[7] M. P. Hansen and A. Jaszkiewicz, "Evaluating the quality of approximations of the non-dominated set," Technical University of Denmark, Tech. Rep. IMM-REP-1998-7, 1998.

[8] E. Zitzler, J. Knowles, and L. Thiele, "Quality assessment of pareto set approximations," in Multiobjective Optimization: Interactive and Evolutionary Approaches, J. Branke, K. Deb, K. Miettinen, and R. Slowinski, Eds. Springer, 2008.

[9] D. Brockhoff, T. Wagner, and H. Trautmann, "On the properties of the R2 indicator," in Proc. ACM Int'l Genetic and Evol. Computat. Conference, 2012.

[10] E. Zitzler, L. Thiele, and J. Bader, "SPAM: Set preference algorithm for multiobjective optimization," in Proc. of Int'l Conference on Parallel Problem Solving From Nature, 2008.
[11] H. Trautmann, T. Wagner, and D. Brockhoff, "R2-EMOA: Focused multiobjective search using R2-indicator-based selection," in Proc. Learning and Intelligent Optimization Conference, Jan. 2013.

[12] Q. Zhang and H. Li, "MOEA/D: A multiobjective evolutionary algorithm based on decomposition," IEEE Trans. Evol. Computat., vol. 11, no. 6, 2007.

[13] K. Deb, A. Pratap, S. Agarwal, and T. Meyarivan, "A fast and elitist multiobjective genetic algorithm: NSGA-II," IEEE Trans Evol. Computat., vol. 6, no. 2, 2002.

[14] E. Zitzler and L. Thiele, "Multiobjective optimization using evolutionary algorithms: A comparative study," in Proc. Int'l Conf. on Parallel Problem Solving from Nature, 1998.

[15] N. Beume, C. Fonseca, M. Lopez-Ibanez, L. Paquete, and J. Vahrenhold, "On the complexity of computing the hypervolume indicator," IEEE Trans. Evol. Computat., vol. 13, no. 5, 2009.

[16] J. Bader and E. Zitzler, "HypE: An algorithm for fast hypervolumebased many-objective optimization," Evol. Computat., vol. 19, no. 1, 2011.

[17] A. Auger, J. Bader, D. Brockhoff, and E. Zitzler, "Theory of the hypervolume indicator: optimal $\mu$-distributions and the choice of the reference point," in Proc. ACM SIGEVO Workshop on Foundations of Genetic Algorithms, 2009.

[18] K. Deb, K. Sindhya, and T. Okabe, "Self-adaptive simulated binary crossover for real-parameter optimization," in Proc. of ACM Int'l Genetic and Evolutionary Computation Conference, 2007.

[19] E. Zitzler, K. Deb, and L. Thiele, "Comparison of multiobjective evolutionary algorithms: Empirical results," Evol. Computat., vol. 8, no. 2, 2000.

[20] K. Deb, L. Thiele, M. Laumanns, and E. Zitzler, "Scalable test problems for evolutionary multiobjective optimization," in Evolutionary Multiobjective Optimization, A. Abraham, R. Jain, and R. Goldberg, Eds. Springer, 2005.

[21] J. Durillo, A. Nebro, and E. Alba, "The jMetal framework for multiobjective optimization: Design and architecture," in Proc. of IEEE Congress on Evolutionary Computation, 2010. 
TABLE III: Comparison of R2-IBEA with Four Other EMOAs in DTLZ Problems with Three Objectives. * and \# are placed when a result is statistically different from R2-IBEA's result based on a $t$-test or a WMW test, respectively.

\begin{tabular}{|c|c|c|c|c|c|c|}
\hline Problem & Algorithm & HVR & GS & GD & IGD & Epsilon \\
\hline \multirow{5}{*}{ DTLZ1 } & R2-IBEA & $\overline{\mathbf{0 . 9 5 1 9 6}(8.7 \mathrm{E}-4)}$ & $\overline{\mathbf{0 . 1 7 8 0 8}(0.0159)}$ & 7.2E-4(4.0E-5) & 4.6E-4(1.0E-5) & $\overline{\mathbf{0 . 0 3 2 2 6}}(0.00103)$ \\
\hline & NSGAII & $0.85918(0.1897)^{* \#}$ & $0.6706(0.36774)^{* \#}$ & $0.06438(0.17935) \#$ & $9.7 \mathrm{E}-4(0.00129) \#$ & $0.07303(0.04873)^{* \#}$ \\
\hline & MOEA/D & $0.9077(0.00329) * \#$ & $0.62114(0.09202)^{* \#}$ & $0.00515(0.01165) \#$ & $5.7 \mathrm{E}-4(1.0 \mathrm{E}-5)^{* \#}$ & $0.0516(0.00182)^{* \#}$ \\
\hline & IBEA- $\varepsilon 2$ & $0.27685(0.1058)^{* \#}$ & $1.81149(0.04458)^{* \#}$ & $0.00331(0.00719) \#$ & $0.00392(3.7 \mathrm{E}-4)^{* \#}$ & $0.27813(0.02383)^{* \#}$ \\
\hline & R2-EMOA & $0.67877(0.11786)^{* \#}$ & $0.98102(0.20714)^{* \#}$ & $0.01045(0.01488)^{* \#}$ & $0.00224(6.0 \mathrm{E}-4)^{* \#}$ & $0.18275(0.04582)^{* \#}$ \\
\hline \multirow{5}{*}{ DTLZ2 } & R2-IBEA & $\mathbf{0 . 9 0 1 9 7}(0.00114)$ & $\mathbf{0 . 2 5 3 7 8}(0.01696)$ & 7.0E-4(1.0E-5) & $7.9 \mathrm{E}-4(3.0 \mathrm{E}-5)$ & 0.06389 $(0.00312)$ \\
\hline & NSGAII & $0.79904(0.01341)^{* \#}$ & $0.50246(0.04331)^{* \#}$ & $0.00136(1.5 \mathrm{E}-4)^{* \#}$ & $7.7 \mathrm{E}-4(5.0 \mathrm{E}-5)$ & $0.13122(0.01821)^{* \#}$ \\
\hline & MOEA/D & $0.78334(0.00366)^{* \#}$ & $0.65507(0.01381)^{* \#}$ & $8.0 \mathrm{E}-4(3.0 \mathrm{E}-5)^{*} \#$ & 7.5E-4 $(0.0)^{* \#}$ & $0.15653(0.00526)^{* \#}$ \\
\hline & IBEA- $\varepsilon 2$ & $0.89703(0.0013) * \#$ & $0.5116(0.02287)^{* \#}$ & $8.0 \mathrm{E}-4(2.0 \mathrm{E}-5)^{* \#}$ & $0.00101(3.0 \mathrm{E}-5)^{* \#}$ & $0.07553(0.00464)^{* \#}$ \\
\hline & R2-EMOA & $0.84573(0.02044) * \#$ & $0.69381(0.07312)^{* \#}$ & 7.4E-4(5.0E-5)*\# & $0.00112(1.3 \mathrm{E}-4)^{* \#}$ & $0.1222(0.02498)^{* \#}$ \\
\hline \multirow{5}{*}{ DTLZ3 } & R2-IBEA & $0.88035(()$ & 0.276 & $\overline{0.00141(2.7 \mathrm{E}-4)}$ & $\overline{\mathbf{0 . 0 0 1 2 4}(4.0 \mathrm{E}-5)}$ & 0.0712( \\
\hline & NSGAII & $0.53293(0.29057)^{* \#}$ & $0.67846(0.25043)^{* \#}$ & $0.04703(0.08537)^{* \#}$ & $0.00397(0.00519)^{* \#}$ & $0.28068(0.25895)^{* \#}$ \\
\hline & MOEA/D & $0.77583(0.01864)^{* \#}$ & $0.73502(0.05477)^{* \#}$ & $0.00174(7.8 \mathrm{E}-4)$ & $0.00128(3.0 \mathrm{E}-5)^{* \#}$ & $0.15637(0.01016)^{* \#}$ \\
\hline & IBEA- $\varepsilon 2$ & $2.3 \mathrm{E}-4(0.00104)^{* \#}$ & $1.17545(0.26827)^{* \#}$ & $0.00208(0.00289)$ & $0.00886(8.0 \mathrm{E}-5)^{* \#}$ & $0.42664(0.00354)^{* \#}$ \\
\hline & R2-EMOA & $0.39757(0.19708)^{* \#}$ & $1.03513(0.22443)^{* \#}$ & $0.05035(0.10598) \#$ & $0.00613(0.00489)^{* \#}$ & $0.4222(0.32368)^{* \#}$ \\
\hline \multirow{5}{*}{ DTLZ4 } & R2-IBEA & $\mathbf{0 . 9 3 7 9 8}(0.15235)$ & $\overline{\mathbf{0 . 3 1 0 3 7}}(0.13908)$ & $0.0055(3.8 \mathrm{E}-4)$ & $0.00169(0.00202)$ & $0.11369(0.1771)$ \\
\hline & NSGAII & $0.88922(0.01346) \#$ & $0.48302(0.0425)^{* \#}$ & $0.00522(3.4 \mathrm{E}-4) * \#$ & 0.00117(1.0E-4)\# & $\mathbf{0 . 1 1 3 6 2}(0.01832) \#$ \\
\hline & MOEA/D & $0.79323(0.16151)^{* \#}$ & $0.73279(0.10628)^{* \#}$ & $0.0073(7.1 \mathrm{E}-4)^{* \#}$ & $0.00244(0.00239) \#$ & $0.23803(0.20247)^{* \#}$ \\
\hline & IBEA- $\varepsilon 2$ & $0.80832(0.23937)^{* \#}$ & $0.64507(0.14167)^{* \#}$ & $0.00659(6.8 \mathrm{E}-4)^{* \#}$ & $0.00278(0.0025)$ & $0.26589(0.27536)^{* \#}$ \\
\hline & R2-EMOA & $0.55039(0.27056)^{* \#}$ & $0.87632(0.07351)^{* \#}$ & $0.00317(0.0013)^{* \#}$ & $0.00522(0.00285)^{* \#}$ & $0.53495(0.27785)^{* \#}$ \\
\hline \multirow{5}{*}{ DTLZ7 } & R2-IBEA & $\mathbf{0 . 9 3}(0.00386)$ & 0.31806(0.02857) & $0.00123(6.0 \mathrm{E}-5)$ & $\mathbf{0 . 0 0 1 9 3}(2.0 \mathrm{E}-4)$ & $\overline{\mathbf{0 . 0 5 8 8 7}}(0.00466)$ \\
\hline & NSGAII & $0.86509(0.01266)^{* \#}$ & $0.48333(0.04511)^{* \#}$ & $0.00297(4.7 \mathrm{E}-4) * \#$ & $0.00224(1.7 \mathrm{E}-4)^{* \#}$ & $0.12314(0.01913)^{* \#}$ \\
\hline & MOEA/D & $0.80663(0.00278)^{* \#}$ & $1.11576(0.014)^{* \#}$ & $0.00126(4.0 \mathrm{E}-5) *$ & $0.00382(8.0 \mathrm{E}-5)^{* \#}$ & $0.17583(0.01388)^{* \#}$ \\
\hline & IBEA- $\varepsilon 2$ & $0.92278(0.00651)^{* \#}$ & $0.46575(0.04426)^{* \#}$ & $0.00121(6.0 \mathrm{E}-5)$ & $0.00318(1.7 \mathrm{E}-4)^{* \#}$ & $0.07004(0.00393)^{* \#}$ \\
\hline & R2-EMOA & $0.81508(0.06628)^{* \#}$ & $0.66229(0.08843)^{* \#}$ & $0.00149(2.1 \mathrm{E}-4)^{* \#}$ & $0.00557(0.0053)^{* \#}$ & $0.60422(0.38011)^{* \#}$ \\
\hline
\end{tabular}

[22] D. A. V. Veldhuizen and G. B. Lamont, "Multiobjective evolutionary algorithm test suites," in Proc. ACM Symposium on Appl. Comput., 1999.

[23] A. Zhou, Y. Jin, Q. Zhang, B. Sendhoff, and E. Tsang, "Combining model-based and genetics-based offspring generation for multi-objective optimization using a convergence criterion," in Proc. IEEE Congress on Evolutionary Computation, 2006.

[24] D. A. V. Veldhuizen and G. B. Lamont, "Multiobjective evolutionary algorithm research: A history and analysis," in TR-98-03, Air Force Institute of Technology, 1998.

[25] E. Zitzler, L. Thiele, M. Laumanns, C. M. Fonseca, and V. G. da Fonseca, "Performance assessment of multiobjective optimizers: an analysis and review," IEEE Trans. Evol. Computat., vol. 7, no. 2, 2003.
TABLE IV: Comparison of R2-IBEA with Four Other EMOAs in DTLZ problems with Five Objectives. * and \# are placed when a result is statistically different from R2-IBEA's result based on a $t$-test or a WMW test, respectively.

\begin{tabular}{|c|c|c|}
\hline Problem & Algorithm & $\mathrm{HV}$ \\
\hline \multirow{5}{*}{ DTLZ1 } & R2-IBEA & $\mathbf{0 . 9 5 2 2 9}(9.2 \mathrm{E}-4)$ \\
\hline & NSGAII & $0.0(0.0) * \#$ \\
\hline & MOEA/D & $0.84326(0.03249)^{* \#}$ \\
\hline & IBEA- $\varepsilon 2$ & $0.35529(0.1649)^{* \#}$ \\
\hline & R2-EMOA & $0.23187(0.23548)^{*} \#$ \\
\hline \multirow{5}{*}{ DTLZ2 } & R2-IBEA & $0.66312(0.00321)$ \\
\hline & NSGAII & $0.19794(0.04368)^{* \#}$ \\
\hline & MOEA/D & $0.43481(0.00453)^{*} \#$ \\
\hline & IBEA- $\varepsilon 2$ & $\mathbf{0 . 6 6 8 4 1}(0.00143)^{* \#}$ \\
\hline & R2-EMOA & $0.57899(0.01613)^{* \#}$ \\
\hline \multirow{5}{*}{ DTLZ3 } & R2-IBEA & 0.62426(0.01833) \\
\hline & NSGAII & $0.0(0.0) * \#$ \\
\hline & MOEA/D & $0.16932(0.11391)^{* \#}$ \\
\hline & IBEA- $\varepsilon 2$ & $0.00118(0.00235)^{* \#}$ \\
\hline & R2-EMOA & $0.0(0.0) * \#$ \\
\hline \multirow{5}{*}{ DTLZ4 } & R2-IBEA & $0.6605(0.02121)$ \\
\hline & NSGAII & $0.23244(0.07753)^{*} \#$ \\
\hline & MOEA/D & $0.41795(0.02613)^{* \#}$ \\
\hline & IBEA- $\varepsilon 2$ & $0.65121(0.04147) \#$ \\
\hline & R2-EMOA & $0.44429(0.07888)^{*} \#$ \\
\hline \multirow{5}{*}{ DTLZ7 } & R2-IBEA & 0.20674(0.00418) \\
\hline & NSGAII & $0.07111(0.01347)^{* \#}$ \\
\hline & MOEA/D & $0.11086(0.01103)^{* \#}$ \\
\hline & IBEA- $\varepsilon 2$ & $0.20075(0.00607)^{* \#}$ \\
\hline & R2-EMOA & $0.10087(0.01433)^{*} \#$ \\
\hline
\end{tabular}




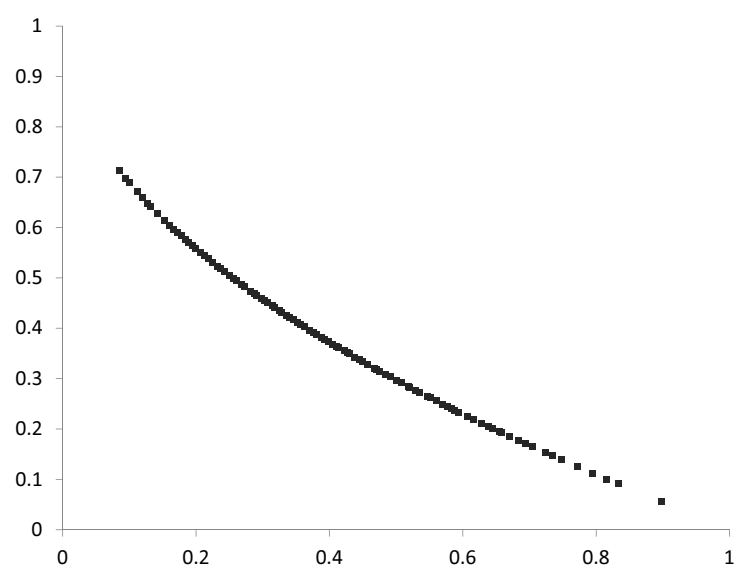

(a) R2-IBEA w/o adaptive reference point selection (ZDT4)

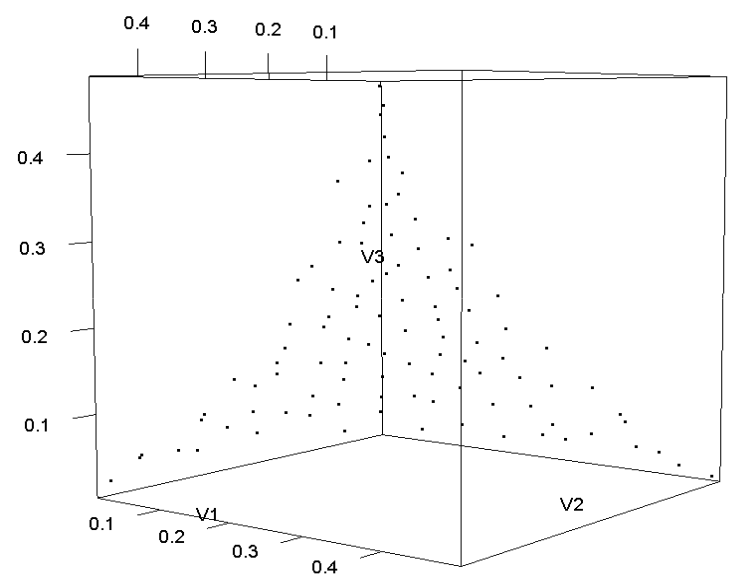

(c) R2-IBEA w/o adaptive reference point selection (DTLZ1)

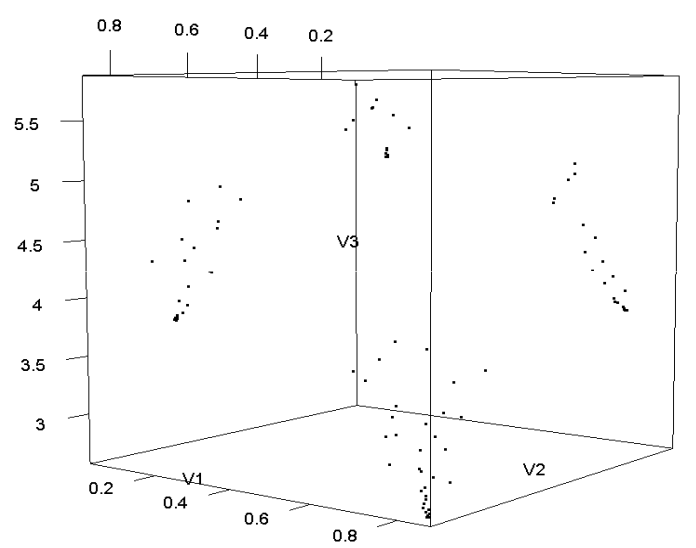

(e) R2-IBEA w/o adaptive reference point selection (DTLZ7)

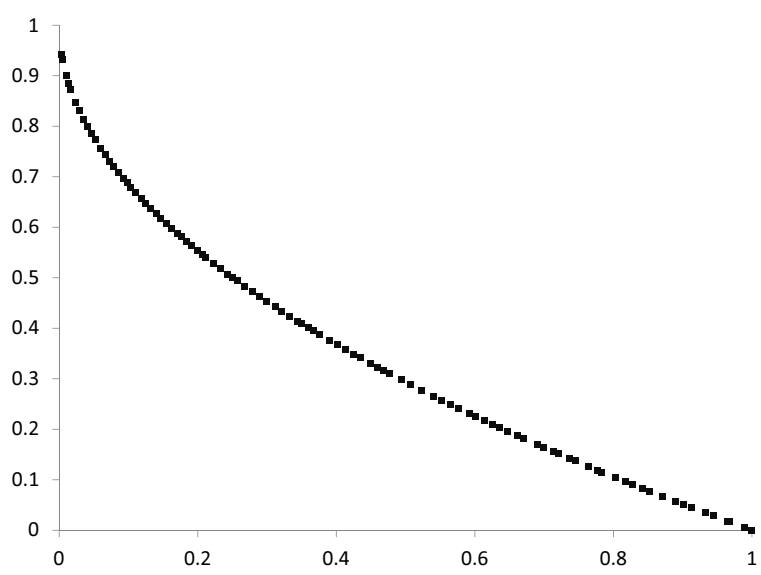

(b) R2-IBEA with adaptive reference point selection (ZDT4)

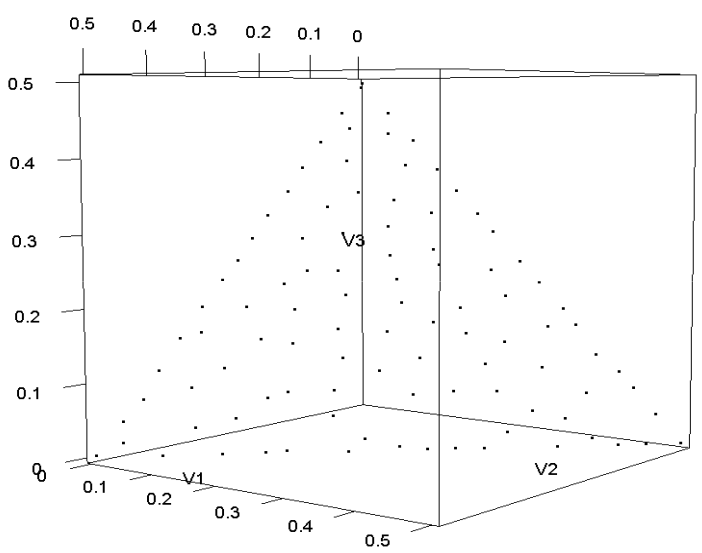

(d) R2-IBEA with adaptive reference point selection (DTLZ1)

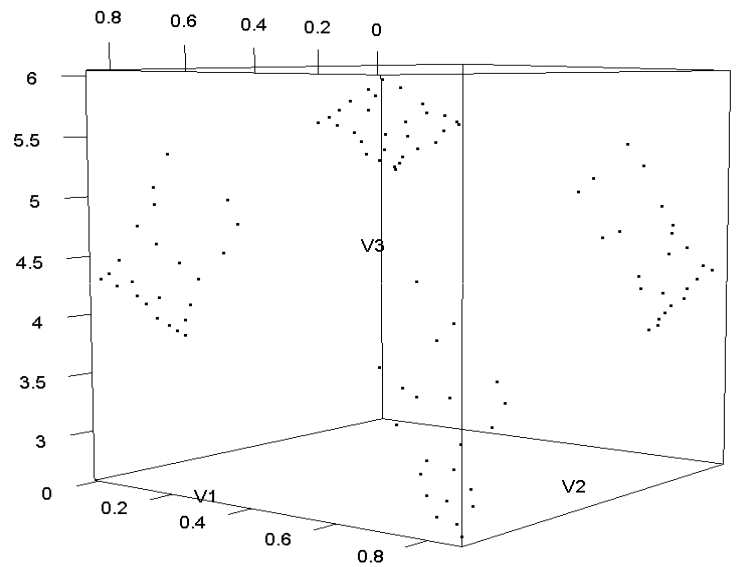

(f) R2-IBEA with adaptive reference point selection (DTLZ7)

Fig. 3: Pareto-approximated individuals in ZDT4, DTLZ1 and DTLZ7 


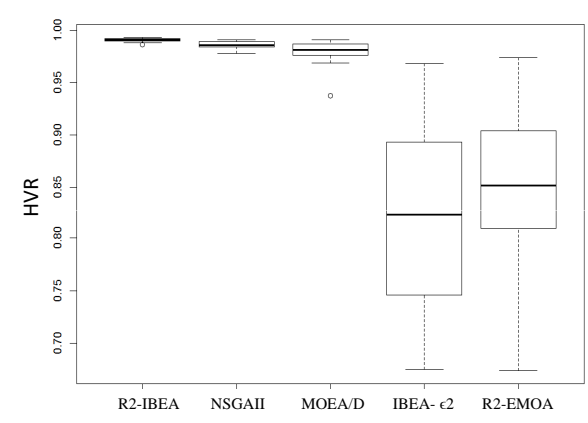

(a) HVR in ZDT4

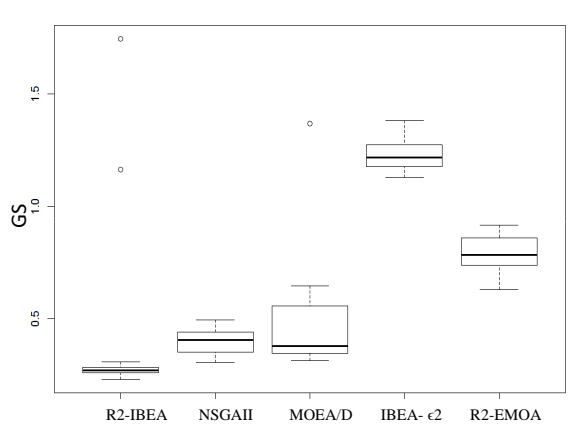

(d) GS in ZDT4

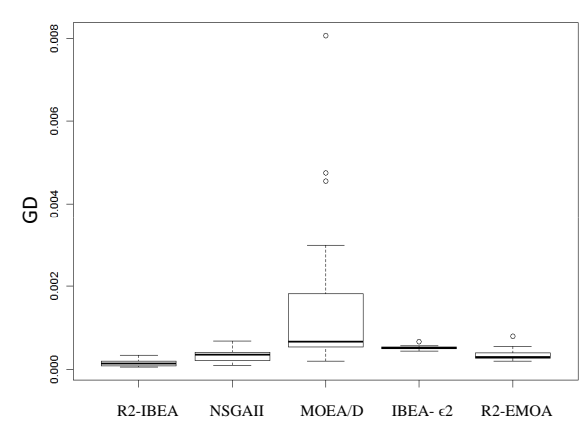

(g) GD in ZDT4

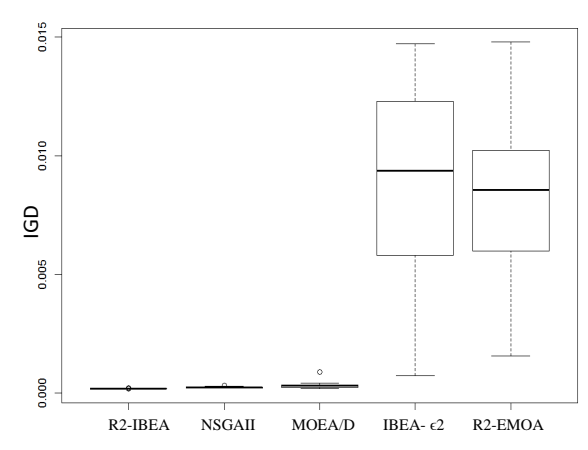

(j) IGD in ZDT4

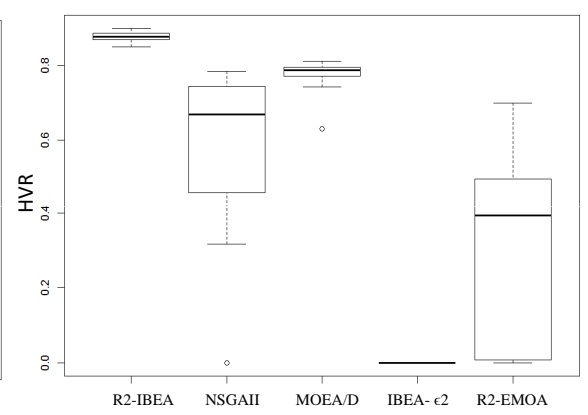

(b) HVR in DTLZ3

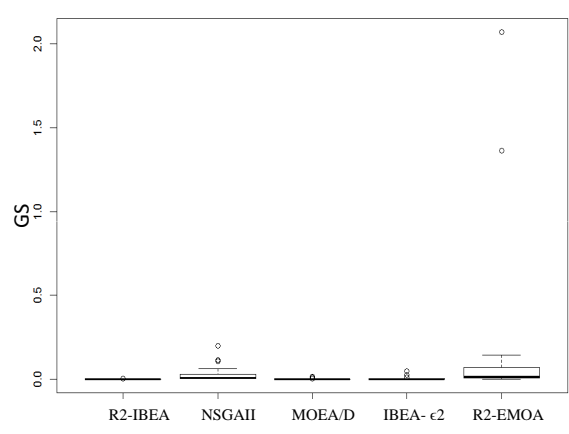

(e) GS in DTLZ3

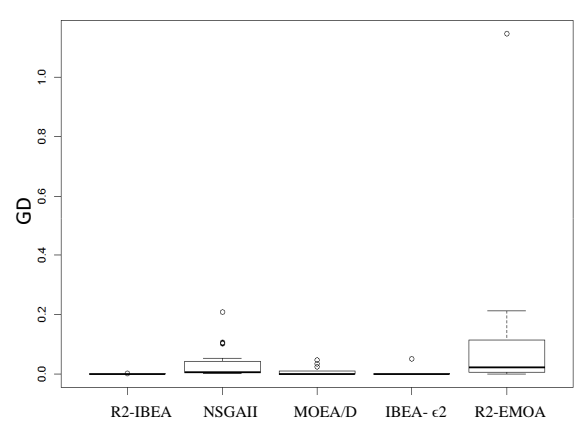

(h) GD in DTLZ3

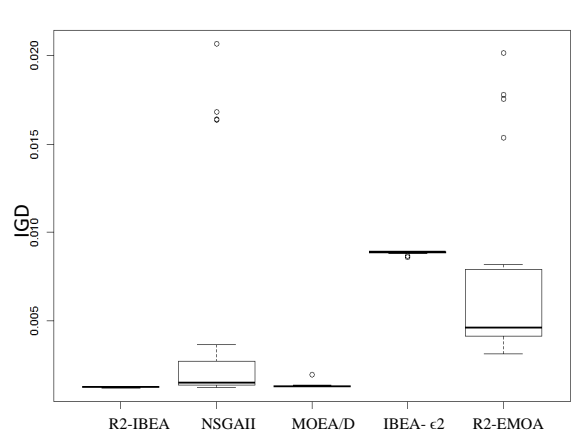

(k) IGD in DTLZ3

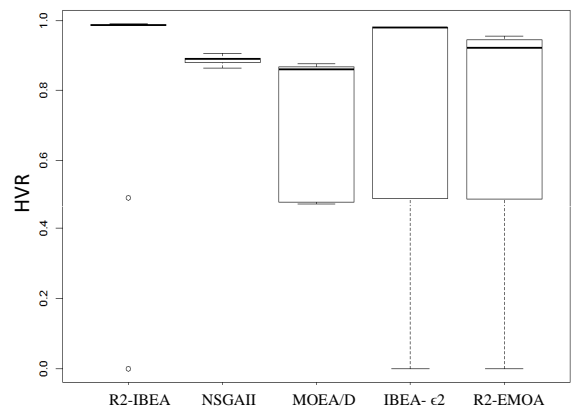

(c) HVR in DTLZ4

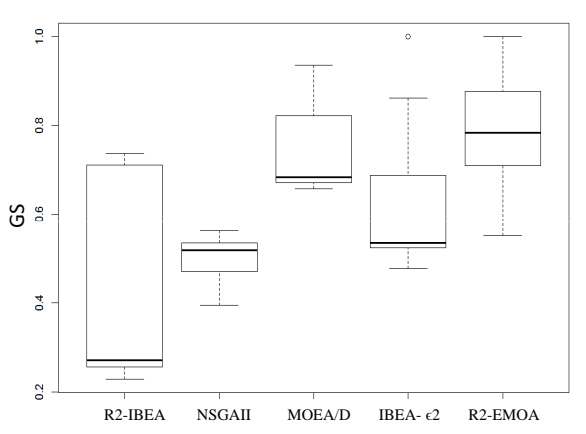

(f) GS in DTLZ4

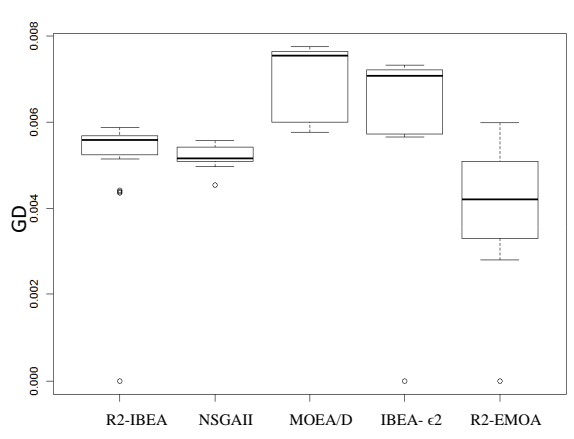

(i) GD in DTLZ4

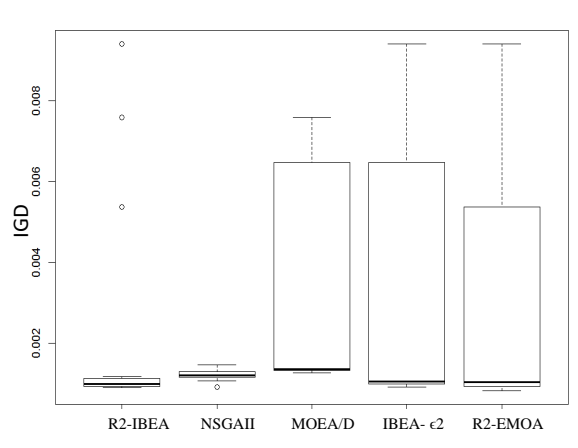

(1) IGD in DTLZ4

Fig. 4: Boxplots for ZDT4, DTLZ3 and DTLZ4 